\title{
Preparing for the Future
}

\author{
Jerris R. Hedges, MD, MS
}

\author{
Oregon Health Sciences University, \\ Department of Emergency Medicine, \\ Portland, Oregon \\ Presented at the 1994 Annual Meeting of \\ the National Association of Emergency \\ Medical Services Physicians, Portland \\ Oregon, August 1994 \\ Dr. Hedges is the Editor-in-Chief of \\ Academic Emergency Medicine, the official \\ medical journal of the Society of \\ Academic Emergency Medicine. \\ Correspondence: Jerris R. Hedges, MD, MS, \\ OHSU, Department of Emergency \\ Medicine, 3181 SW Sam Jackson Park \\ Road, UHN-52, Portland, OR 97201-3098 \\ USA \\ Acknowledgments: Discussions with Ronald \\ Maio, DO of the University of Michigan, \\ and my OHSU colleagues have con- \\ tributed significantly to this essay.
}

Key Words: emergency medical services, managed care
Abbreviations:
$\mathrm{CHF}=$ congestive heart failure
$\mathrm{ED}=$ emergency department
EMS = emergency medical services
SVT = supraventricular tachycardia

I will address briefly the future of emergency medical services (EMS) and the role of EMS research in that future.

The foreseeable future of health care will be dominated by managed-care systems that will limit health-care funding. These systems are evolving earlier in several areas of the country. It is ironic that Minneapolis, Minnesota, and Portland, Oregon, the sites of the last two National Association of EMS Physicians annual meetings, are on the leading edge of the managed-care movement.

Keeping things in simple terms, I see three general themes in managed care.

1) less pay for medical services;

2) consolidation of medical providers; and

3) health care administrated on a population-based model.

First, how each of these general themes impacts EMS will be addressed briefly, and then the role of research in this environment will be discussed.

\section{Impact on EMS}

How will "less pay for medical services" affect EMS systems? Primarily, insurance companies providing managed care will negotiate in advance for a fixed annual sum to provide EMS care for their patient population. This will limit funds available for physician supervision, research, and clinical innovations.

How will consolidation of medical providers affect EMS systems? Basically, hospitals, clinics, and physician providers will network and merge their services. Ambulance destinations for patients will be determined by contractual arrangements that the providers have made with the insurers (sometimes the providers will be the insurers). The general concept of regional health resources (e.g., the trauma center concept) will be challenged as major medical provider organizations begin to clash for total control of their patients (or more correctly, total control over the cost of health care for their insured patients).

How will a population-based model for health care affect EMS systems? Basically, decisions regarding clinical care will be shifted from the needs of the individual to the needs of the community. However, the community will be defined as those who are covered by major insurers.

The needs of the indigent population may be ignored in policy decisions by major insurers unless a governmental health plan is developed that adequately pays for their needs. This governmental involvement is the driving force of the Oregon Health Plan. However, the individual provider will remain responsible for the individual patient, although the provider will be expected to provide care under rules developed for the masses rather than tailored for the individual.

Is this a doom-and-gloom scenario? I really don't think so. Our current healthcare system has considerable problems: 1)expensive therapies are provided to a select few; 2) the health-care industry is encouraged to treat illness and injury, but does not account for its expenditures, nor does it necessarily promote health; and 3)health-care rationing is a way of life for the poor, but currently is denied by insurers and other administrators. These problems will be attacked under managed care. If EMS systems truly provide valued and effective services to a large population of patients for a reasonable cost, justification for increased support can be made.

\section{Role of Research}

I believe that EMS systems will not just survive in the future, but will thrive. However, they must adjust to this new practice environment. Population-based EMS research can help. Your EMS system's database can show health-care planners which services people want. Scientific analysis of EMS outcomes can help deter- 
mine which services people need.

Our task as researchers and physician supervisors is to educate those who are emerging as the brokers in the population-based health-care arena as to the true value of using the EMS system:

1) monitor communitywide health-care use; 1

2) identify potential injury and illness preventative measures; ${ }^{1}$ and

3) provide some health care entirely out-of-hospital.

We will need to demonstrate cost-effectiveness in our patient assessments and interventions. This will require us to monitor "customer satisfaction" and will challenge traditional EMS care practices. For example, much of EMS is focused on getting patients to the emergency department (ED), but often patient care should begin and end at the scene ... entirely out of the hospital.

\section{Out-of-Hospital Care}

Fourteen years ago when I supervised the Thrust County Medic One Program in Washington, we established individualized, out-of-hospital care protocols for many complex patients who commonly required ED evaluation and hospital admission. A couple of examples that may guide future cost-effective analyses should be valuable.

One such patient had end-stage chronic congestive heart failure (CHF) and lived approximately 40 minutes from the hospital. She was seen in the ED and admitted several times each month for her CHF. The patient and her family preferred that she not be treated further in the hospital. However, the patient did want further treatment for her CHF episodes when they did occur.

With her primary-care provider, we developed a care plan for home treatment. The patient received oxygen (and bag-valve-mask ventilatory support as needed), intravenous furosemide, intravenous morphine as needed for undue anxiety, and nitroglycerin paste.

During an exacerbation, the patient was managed for 30 to 60 minutes at her home. If she was improving, the paramedics continued to provide care until they believed she was stabilizing, and then they left the patient at home. If the patient was considered to need additional therapy, medical command was contacted and options discussed. Her visits to the ED and corresponding hospitalizations dropped to almost none. When the patient did die after more than a year of intermittent home EMS treatments, her death occurred at home where she chose it to occur.

Another frequent ED patient had recurrent bouts of supraventricular tachycardia (SVT) requiring pharmacologic conversion every few months in the ED. A treatment protocol was developed permitting paramedics to use intravenous verapamil for known SVT patients who had responded to this treatment in the past. This patient and others were converted pharmacologically out-of-hospital, observed by the paramedics, and then released in the absence of chest discomfort, pulmonary edema, or hypotension. Of note, this approach was adopted years before adenosine became available.

The future practice environment requires that physician supervisors develop clear guidelines for the treatment and release of patients out-of-hospital. In present EMS systems, I suspect that hypoglycemic diabetic patients and previously diagnosed seizure patients commonly are treated and released at the scene. ${ }^{2,3}$ However, in many systems this requires a lengthy against medical advice process and perhaps even contact with on-line medical command. ${ }^{3}$

\section{The Future}

Why is all this necessary? Can't we develop guidelines that permit and even encourage release of the patient under specific conditions? ${ }^{4}$ Can't we become partners with primary providers and develop unique plans for specific patients that permit care usually reserved for the ED to be provided at the patient's home?

These concepts have associated medicolegal issues, but a different practice paradigm is feasible. After all, EMS physician supervisors should determine the standard of care through clinical evaluation of practice innovations. Hence, the future will be exciting for EMS and EMS research. Today's research is tomorrow's practice.

\section{References}

1. Hsiao AK, Hedges JR: Role of the emergency medical services system in regionwide health monitoring and referral. Ann Emerg Med 1993;22:1696-1702

2. Stark G, Hedges JR, Neely K, et al: Patients who refuse prehospital evaluation and/or therapy. Am JEmerg Med 1990;8:509-511.

3. Heiser JM, Norton RL, Neely KW, et al: Paramedic on-scene time for patients initially refusing treatment or transport. Prehospital and Disaster Medicine 1991;6:293-297.

4. Thompson RH, Wolford RW: Development and evaluation of criteria allowing paramedics to treat and release patients presenting with hypoglycemia: A retrospective study. Prehospital and Disaster Medicine $1991 ; 6: 309-313$ 